\title{
Experimental validation of a bearing wear model using the directional response of the rotor-bearing system
}

\author{
TIAGO HENRIQUE MACHADO and KATIA L. CAVALCA \\ Laboratory of Rotating Machinery, Faculty of Mechanic Engineering, UNICAMP, Rua \\ Mendeleyev, 200, Postal Box 6122, 13083-970 Campinas, SP, Brazil
}

Manuscript received on June 10, 2015; accepted for publication on November 27, 2015

\begin{abstract}
The present work gives continuity in the analysis of the wear influence on cylindrical hydrodynamic bearings by presenting an experimental validation of the wear model previously proposed by the authors. This validation is carried on using the frequency response of the rotor-bearings system in directional coordinates. For this purpose, a test rig was assembled in order to evaluate the behavior of the rotating system when supported by hydrodynamic bearings with different wear patterns. The experimental measurements are used to validate the wear model, comparing the anisotropy influence on the experimental and numerical responses. The simulated directional frequency responses showed a good agreement with the experimental ones, demonstrating the potential of the proposed wear model in satisfactorily represent its influence on the rotor-bearings system response in the frequency range where the numerical model was validated.
\end{abstract}

Key words: Hydrodynamic bearing, wear model, experimental validation, directional frequency response.

\section{INTRODUCTION}

Journal bearings play an important role in the dynamics of rotor-bearings systems, being responsible for supporting the machine and transmit forces between the rotor and the foundation. For this reason, in order to perform a dynamic analysis of the complete rotating system, it is necessary to know the dynamic characteristics of bearings, understanding the main phenomena related to this component, allowing the prediction of malfunctions or even a proper predictive maintenance.

The operation of a rotor for a long period of time as well as the many starts and stops to which the system is subjected, may result in direct contact between the shaft and the bearing, leading to high friction in the lubricated contact and consequently the wear of the bearings wall. This wear causes discontinuities or geometric changes in the bearing, which influence the bearing radial clearance (Machado and Cavalca (2015)). Therefore, the wear can directly affect the dynamic behavior and stability of the rotating system.

Correspondence to: Tiago Henrique Machado

E-mail: tiagomh@fem.unicamp.br 
Katzenmeier (1972) was one of the first authors to analyze the effects of wear in hydrodynamic bearings. He quantitatively and qualitatively analyzed several examples of damage due to wear. Mokhtar et al. (1977) experimentally conducted the first studies related to the wear. They measured the changes in diametrical clearance, surface finishing and roundness of bearing caused by the wear. Through the study of the wear location on the bearings, the conclusion was that the sliding motion during the start cycles was the main responsible for the development of the wear, while the stop cycle had a negligible contribution.

After the experimental studies, there was still a need to develop models that could satisfactorily represent the main effects of wear on the static and dynamic characteristics of journal bearings. This assignment was carried on by Dufrane et al. (1983). The authors proposed two models to characterize the wear, both with a non-circular geometry. The first model was based on the concept that the shaft makes a 'print' in the bearing and the second model is based on the assumption of an abrasive wear with an arc larger than the bearing diameter. The second model was later experimentally validated in the study conducted by Hashimoto et al. (1986), where two different hydrodynamic lubrication regimes were also investigates, namely, laminar and turbulent.

In the following decade, the characteristics of noncircular bearings, considering the effects of turbulence and cavitation, were numerically analyzed by Vaidyanathan and Keith (1991), for four different bearing profiles, circular, worn-circular, two lobe, and elliptical. At the same year, Scharrer et al. (1991) presented a research on the dynamic coefficients of journal bearings, showing that small wear depths have small influence on bearing performance. Later, Suzuki and Tanaka (1995) analyzed the stability of a worn bearing. They concluded that the wear decreases the stability of the bearing when it is submitted to a light load. The stability of worn bearings was also studied by Kumar and Mishra (1996a, b) in non-laminar lubrication regimes.

Recently, some studies have been published including more effects from the hydrodynamic lubrication in worn bearings. Fillon and Bouyer (2004) presented the thermo-hydrodynamic performance of a worn plain journal bearing. Using the model proposed by Dufrane et al. (1983), a range of wear depth, from 10\% to $50 \%$ of the bearing radial clearance, was tested. The authors concluded that the worn bearing presents not only disadvantages but also some advantages, such as lower temperature. A new theoretical clearance identification method was proposed by Papadopoulos et al. (2008) to be used in rotating systems supported by two hydrodynamic journal bearings. The rotor was modeled using the finite element method and the dynamic coefficients of the bearing were calculated by solving the Reynolds equation. The identification method used as objective function the difference between the measured and calculated properties of the rotor at a particular point. In the same year, Wu et al. (2008) experimentally tracked the friction conditions in hydrodynamic bearing with a system named by the authors as On-Line Ferrograph Visual. They concluded that the predominant wear mechanisms were the micro-plowing and micro-cutting induced by the initial roughness of surfaces in the rotor starting phase.

Some years later, Gertzos et al. (2011) used the computational fluid dynamics (CFD) analysis to solve the Navier-Stokes equations for a worn journal bearing. They also presented a graphical detection method to identify the wear depth associated with the measured dynamic bearing characteristics, proposing, therefore, a real time wear identification. In the same context of time domain analysis, Chasalevris et al. (2013) designed an experimental apparatus to investigate the wear influence on a continuous rotor mounted on worn hydrodynamic bearings. They observed that sub- and super-harmonics are revealed in the continuous wavelet transform (CWT) of the rotor-bearing system response for worn bearings. 
Finally, Machado and Cavalca (2015) presented a numerical model to represent the wear in hydrodynamic bearings. The authors performed an analysis of the model sensitivity to different geometric and operational bearings parameters. Through the proposed model, it was also shown that the wear significantly influences the dynamic response of the rotating system, especially when this response is analyzed in directional coordinates.

Within this context, this paper gives continuity in the analysis of the wear influence on hydrodynamic bearings, presenting an experimental validation of the wear model proposed by Machado and Cavalca (2015). This validation is carried on using the frequency response of the rotor-bearings system in directional coordinates, since, as observed by Machado and Cavalca (2015), this response is significantly influenced by the anisotropy of the system, which increases in the presence of wear. For this purpose, a test rig was assembled in order to evaluate the behavior of the rotating system when supported by hydrodynamic bearings with different wear patterns.

\section{MATERIALS AND METHODS}

The first section refers to the modeling of the complete rotating system, including both modes of the rotor and the bearings. The rotor, modeled by finite elements, is mathematically represented by mass, stiffness and damping matrices. With respect to the bearings, the oil film is represented by equivalent stiffness and damping coefficients, calculated from the pressure distribution obtained by the Reynolds equation. The second part deals with the modeling of the wear and their characteristic parameters, showing how the wear model changes the bearing radial clearance, and consequently, its dynamic characteristic. Finally, the third part consists of calculating the directional frequency response of the rotating system. For this analysis, the rotor-bearings system is modeled in directional coordinates. The physical response of the rotor in the plane normal to the shaft is related to the forward and backward components of the system response.

\section{ROTATING SYSTEM}

The rotating system is modelled by finite elements, through a set of rigid disks and shaft segments with distributed mass and elasticity, and the hydrodynamic bearings, represented by equivalent stiffness and damping coefficients. The analysis is accomplished considering a rigid structure (rigid foundation), in order to highlight the anisotropy effect from the bearing as well as the sensitivity of the rotating system to this effect.

\section{Modelling of the rotor}

The classical equation of motion of the rotating system is defined by most handbooks of rotor dynamics, as Krämer (1993):

$$
[M] \cdot\{\ddot{q}\}+([C]+\Omega \cdot[G]) \cdot\{\dot{q}\}+[K] \cdot\{q\}=\{F\}
$$

where $[\mathrm{M}],[\mathrm{C}],[\mathrm{K}]$ and $[\mathrm{G}]$ are the global mass, damping, stiffness and gyroscopic matrices, respectively. $\{\mathrm{F}\}$ contains the external forces, in the present case, the mass unbalance force.

The [C] matrix, which represents the structural damping of the rotor, is considered proportional to the mass and stiffness matrices (Liu and Novak (1995)), where $\alpha_{C}$ and $\beta_{C}$ coefficients are estimated using the experimental modal analysis of a system in 'free-free' condition: 


$$
[C]=\alpha_{C} \cdot[M]+\beta_{C} \cdot[K]
$$

The Finite Element Model (FEM) is based on the matrices presented in Nelson and McVaugh (1976), considering Timoshenko beam elements, with four degrees of freedom per node: two translational and two rotational. Thereby, the beam elements encompass the translation and rotation inertia effects, gyroscopic moments and elastic bending energy.

\section{Hydrodynamic bearings with geometric discontinuities}

The wear effect is included in the bearing modeling as a geometric discontinuity in the radial clearance of the bearing and, consequently, in the oil film (Figure 1). The procedure, in this paper for the numerical solution of the Reynolds equation, in the specific case of a discontinuous oil film thickness, was introduced by Arghir et al. (2002) in one dimension and expanded to two dimensions in Machado and Cavalca (2011). The approach uses a discretization of the fluid film into a finite volumes mesh (Figure 2).

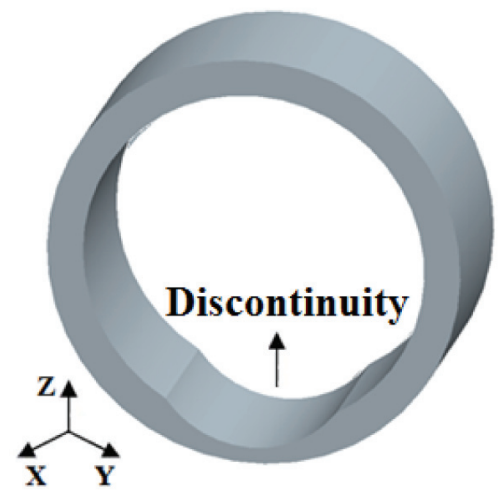

Figure 1 - Bearing with discontinuities in the radial clearance.

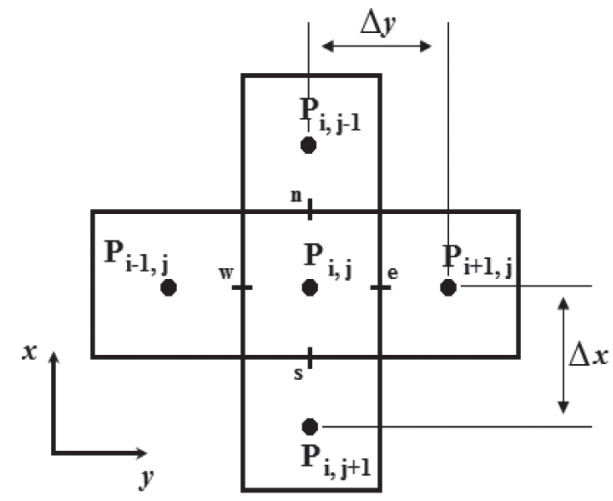

Figure 2 - Finite volume mesh.

According Machado and Cavalca (2011), the hydrodynamic forces components $\mathrm{F}_{\mathrm{r}}$ and $\mathrm{F}_{\mathrm{t}}$ (Figure 3 ) are obtained by the integration of the pressure field. These forces are decomposed in the $\mathrm{Y}-\mathrm{Z}$ inertial system $\left(\mathrm{F}_{\mathrm{y}}\right.$ and $\mathrm{F}_{\mathrm{z}}$ ), using the attitude angle. In order to represent the stiffness $(\mathrm{K})$ and damping $(\mathrm{R})$ coefficients for the bearing (Figure 4), the approximation of equations (4) is used, where $\bar{z}, \bar{y}$ and $\dot{\bar{y}}, \dot{\bar{z}}$ are, respectively, the small perturbations in the shaft displacements and velocities around its equilibrium position at each rotating velocity (Machado and Cavalca (2011)).

$$
\begin{array}{cc}
K_{y y}=\frac{\partial F_{y}}{\partial y} \approx \frac{F_{y+\Delta \bar{y}}-F_{y-\Delta \bar{y}}}{2 \Delta \bar{y}} & K_{y z}=\frac{\partial F_{y}}{\partial z} \approx \frac{F_{y+\Delta \bar{y}}-F_{y-\Delta \bar{y}}}{2 \Delta \bar{z}} \\
K_{z y}=\frac{\partial F_{z}}{\partial y} \approx \frac{F_{z+\Delta \bar{z}}-F_{z-\Delta \bar{z}}}{2 \Delta \bar{y}} & K_{z z}=\frac{\partial F_{z}}{\partial z} \approx \frac{F_{z+\Delta \bar{z}}-F_{z-\Delta \bar{z}}}{2 \Delta \bar{z}} \\
R_{y y}=\frac{\partial F_{y}}{\partial \dot{y}} \approx \frac{F_{y+\Delta \dot{y}}-F_{y-\Delta \dot{y}}}{2 \Delta \dot{\bar{y}}} & R_{y z}=\frac{\partial F_{y}}{\partial \dot{z}} \approx \frac{F_{y+\Delta \dot{y}}-F_{y-\Delta \dot{y}}}{2 \Delta \dot{\bar{z}}} \\
R_{z y}=\frac{\partial F_{z}}{\partial \dot{y}} \approx \frac{F_{z+\Delta \dot{\bar{z}}}-F_{z-\Delta \dot{\bar{z}}}}{2 \Delta \dot{\bar{y}}} & R_{z z}=\frac{\partial F_{z}}{\partial \dot{z}} \approx \frac{F_{z+\Delta \dot{\bar{z}}}-F_{z-\Delta \dot{\bar{z}}}}{2 \Delta \dot{\bar{z}}}
\end{array}
$$




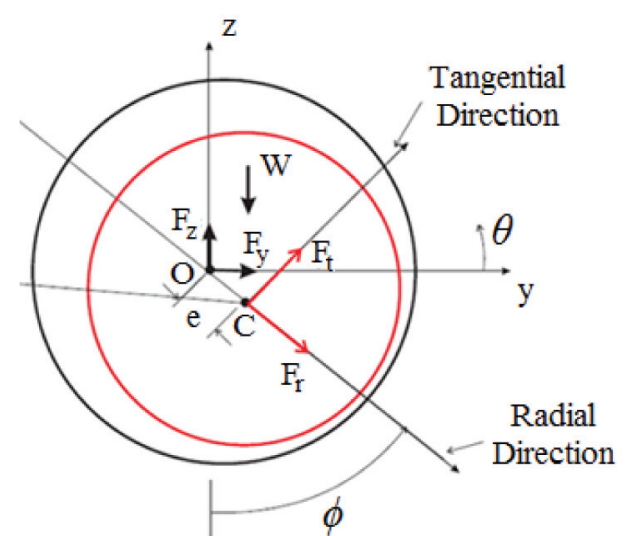

Figure 3 - Schematic representation of the reference system and the forces in the bearing.

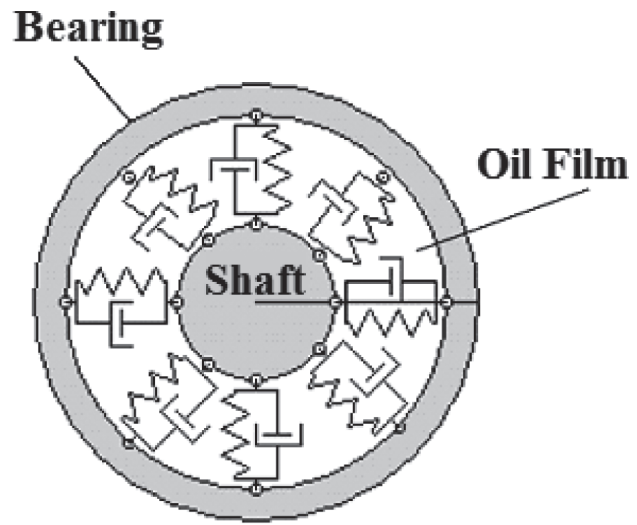

Figure 4 - Mathematical model for the hydrodynamic bearing.

WEAR MODEL

The wear model, proposed by Machado and Cavalca (2015) and used in this paper, is briefly presented here. A schematic worn bearing is shown in Figure 5. This wear pattern introduces an additional oil layer with depth $\delta_{h}(\theta)$ in the region delimited by the angles $\theta_{\mathrm{s}}$ and $\theta_{\mathrm{f}^{\circ}}$ The center of the angular extent of the wear can be displaced by an angular amount relative to the vertical axis, denoted by $\gamma$.

The thickness of the lubricant film in the presence of wear, accordingly to Machado and Cavalca (2015), can be written as:

$$
h(\theta)=h_{0}(\theta)+\delta_{h}(\theta)
$$

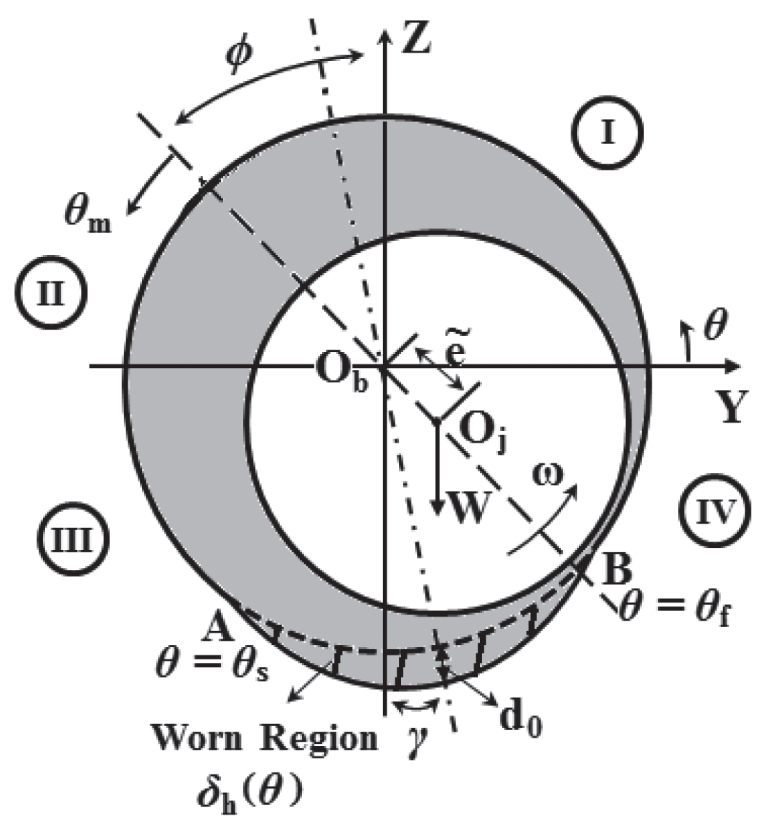

Figure 5 - Worn bearing geometry. 
Where each term can be expressed as:

$$
\begin{gathered}
h_{0}(\theta)=C r-e_{z} \cdot \cos (\theta-\pi / 2)+e_{y} \cdot \operatorname{sen}(\theta-\pi / 2) \\
\delta_{h}(\theta)=d_{0}-C r \cdot(1+\cos (\theta-\pi / 2))
\end{gathered}
$$

where $\mathrm{Cr}$ is the radial clearance. Kwoning that $\delta_{\mathrm{h}}(\theta)$ is delimited by the angles $\theta_{\mathrm{s}}$ and $\theta_{\mathrm{f}}$, the film thickness can be written as follows:

$h(\theta)=\left\{\begin{array}{lc}h_{0}, & 0 \leq \theta \leq \theta_{s}, \quad \theta_{f} \leq \theta \leq 2 \pi \\ h_{0}+\delta_{h}, & \theta_{s}<\theta<\theta_{f}\end{array}\right.$

where the edges of the wear, at the beginning and at the end of the wear region, between $\theta=\theta_{\mathrm{s}}$ and $\theta=\theta_{\mathrm{f}}$, are calculated finding extreme points of the wear, i.e., when depth $\delta_{h}(\theta)$ is null, which leads to:

$\theta_{s}=\pi / 2+\cos ^{-1}\left(d_{0} / C r-1\right)+\gamma$

$\theta_{f}=\pi / 2-\cos ^{-1}\left(d_{0} / C r-1\right)+\gamma$

\section{COMPLEX FORMULATION AND DIRECTIONAL RESPONSE OF THE SYSTEM}

The rotating motion of a rotor shaft consists of two precession components. The direct precession (forward) occurs in the same direction of rotor rotation, and the retrograde precession (backward) occurs in the opposite direction to the rotation of the rotor (Machado and Cavalca (2015)). For a better understanding, an example from Lalanne and Ferraris (1998) is shown in Figure 6, demonstrating the whirl direction for an asymmetric rotor. The response curve is a plot of the maximum amplitude at some point on the rotor's Operation Deflection Shape (ODS).

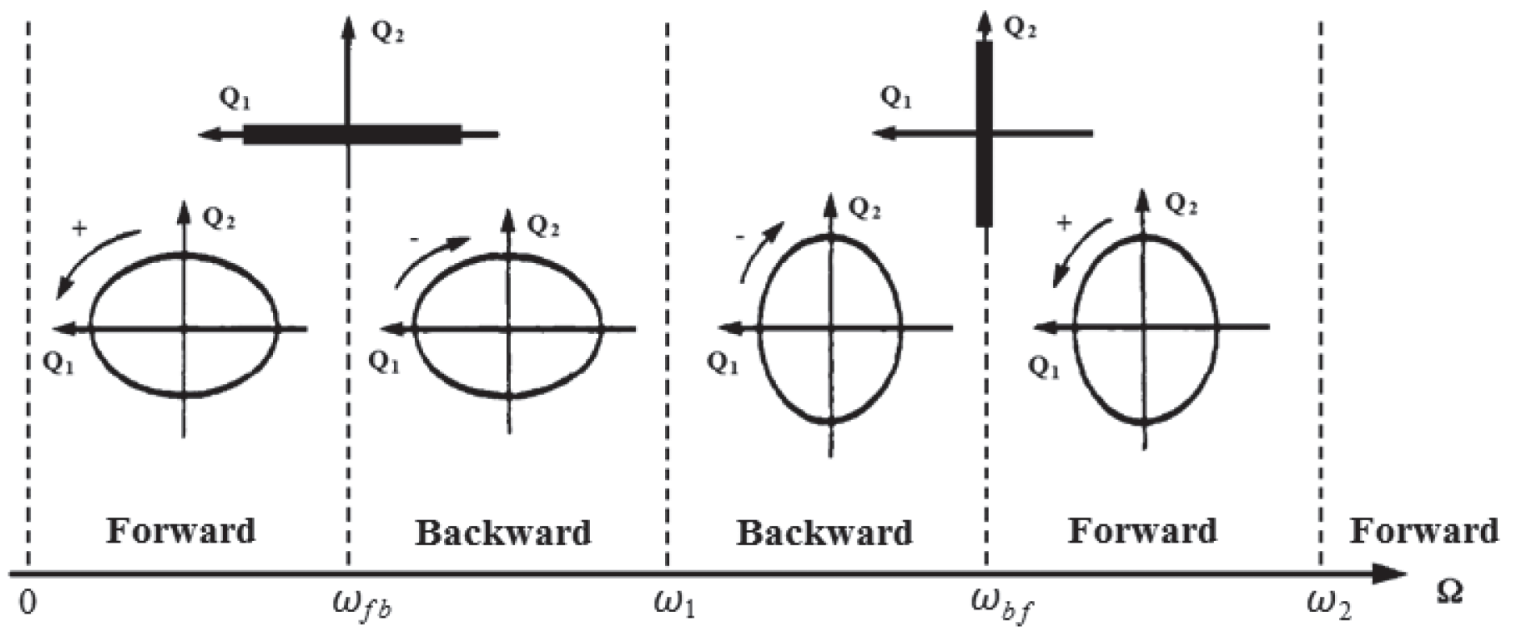

Figure 6 - Variation of the whirl direction for an asymmetric rotor (adapted from Lalanne and Ferraris (1998)). 
In order to do the directional analysis of the frequency response of the rotating system, the rotorbearings system is modeled in directional coordinates. The physical response of the rotor in the y-z plane (perpendicular to the rotor axis) is related to the forward ( $f$ ) and backward (b) components of the system response. The relation between the two coordinate systems was developed by Lee (1991), and was later applied by Machado and Cavalca (2015). The transformation matrix [A] can be defined by equation (9).

$$
\left\{\begin{array}{l}
f \\
\bar{b}
\end{array}\right\}=[A]^{-1}\left\{\begin{array}{l}
y \\
z
\end{array}\right\} ;\left\{\begin{array}{l}
y \\
z
\end{array}\right\}=[A]\left\{\begin{array}{l}
f \\
-b
\end{array}\right\}
$$

where $\bar{b}$ is the complex conjugate of $\mathrm{b}$.

The transformation matrix $[\mathrm{A}]$ and its inverse are expressed as:

$$
[A]=\left[\begin{array}{cc}
1 & 1 \\
-j & j
\end{array}\right] ; \quad[A]^{-1}=\left[\begin{array}{cc}
1 / 2 & j / 2 \\
1 / 2 & -j / 2
\end{array}\right]
$$

The angular and translational coordinates for each of the nodes of the beam elements can be transformed, using the following relations:

$$
\left\{x_{r}\right\}=\left[T_{A}\right]\{p\} ; \quad\{p\}=\left[T_{A}\right]^{-1}\left\{x_{r}\right\}
$$

where the vectors $\left\{\mathrm{x}_{\mathrm{r}}\right\}$ and $\{\mathrm{p}\}$, and the matrices $\left[\mathrm{T}_{\mathrm{A}}\right]$ and $\left[\mathrm{T}_{\mathrm{A}}\right]^{-1}$ are:

$$
\left\{x_{r}\right\}=\left\{\begin{array}{c}
y_{i} \\
z_{i} \\
\alpha_{i} \\
\beta_{i}
\end{array}\right\} ; \quad\{p\}=\left\{\begin{array}{l}
f_{i} \\
\bar{b}_{i} \\
\gamma_{i} \\
\bar{\eta}_{i}
\end{array}\right\} ; \quad\left[T_{A}\right]=\left[\begin{array}{cc}
{[A]} & {[0]} \\
{[0]} & {[A]}
\end{array}\right] ; \quad\left[T_{A}\right]^{-1}=\left[\begin{array}{cc}
{[A]^{-1}} & {[0]} \\
{[0]} & {[A]^{-1}}
\end{array}\right]
$$

In this equation $y_{\mathrm{i}}$ and $z_{\mathrm{i}}$ are the displacements, $\alpha_{\mathrm{i}}$ and $\beta_{\mathrm{i}}$ are the angular coordinates, $f_{\mathrm{i}}$ and $b_{\mathrm{i}}$ are the directional coordinates, $\gamma_{i}$ and $\eta_{i}$ are the directional angular coordinates, all related to the $i$-th node.

\section{EXPERIMENTAL APPARATUS}

The test rig used to obtain the experimental results is presented here, along with the required instrumentation and the description of the experimental procedure.

\section{TEST RIG DESCRIPTION}

The test rig (Figure 7) consists of a grounded inertial concrete base, on which a second concrete block is supported by a set of high stiffness springs and a high density polyurethane foam, providing vibration isolation from the ground. A steel base is clamped to the second concrete block by metallic columns, characterizing a rigid foundation to the rotor-bearing system. 


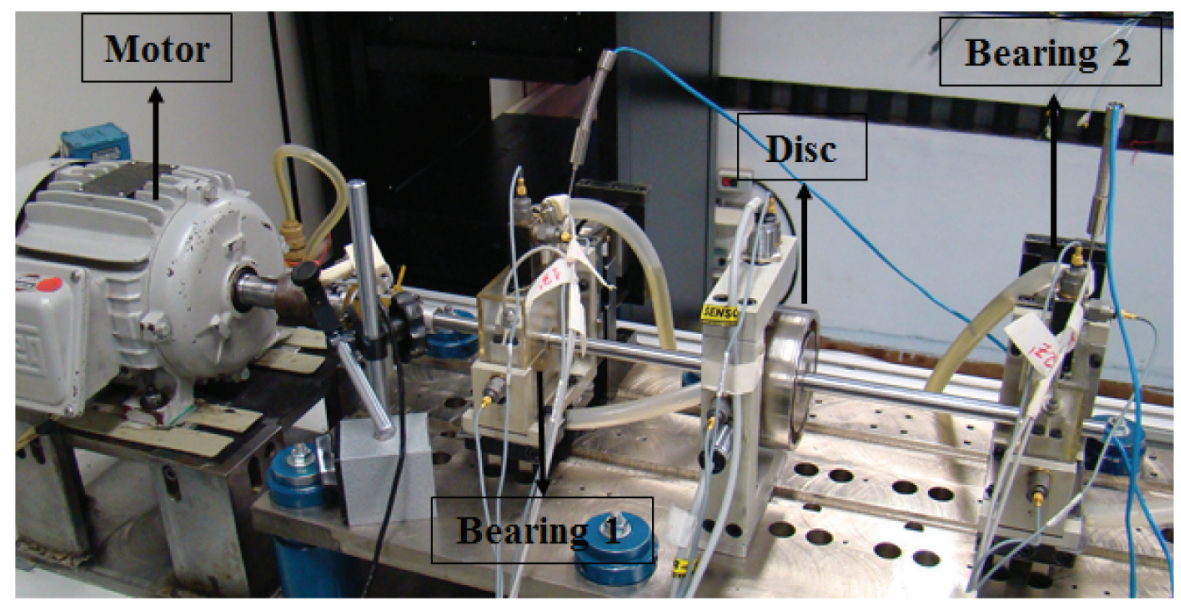

Figure 7 - Experimental test rig.

The rotor-bearing system is composed of a steel shaft, two identical journals coincident to the bearings position, and a stainless steel rigid disc, positioned in the mid span between the bearings. Figure 8 shows a sketch with the components and dimensions of the rotor mounted in the test rig. A trigger or a 'keyphasor' (optical sensor) is assembled close to the shaft-motor coupling in order to set the phase reference for the experiments. The bearings have radial clearance of $90 \mu \mathrm{m}$, width of $20 \mathrm{~mm}$ and inner diameter of $30 \mathrm{~mm}$.

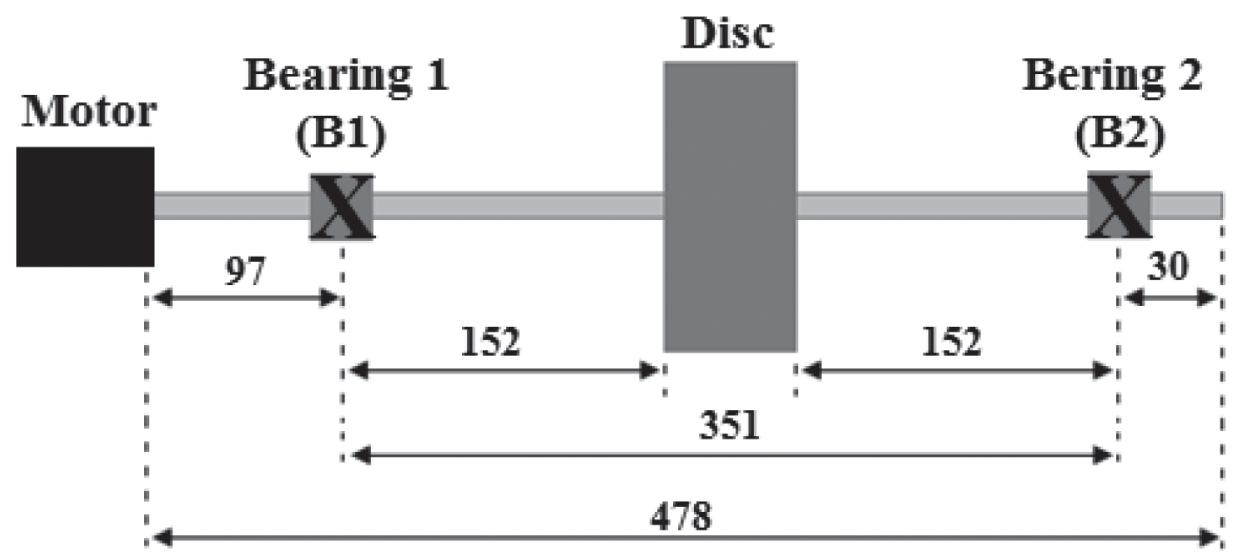

Figure 8 - Sketch of the rotor used in the test rig (dimensions in $\mathrm{mm}$ ).

Oil is pumped from a reservoir to the bearings inlet, passing through a filter and providing a continuous oil supply in the bearings. The oil flowing through the bearing side is then collected and pumped back into the reservoir. The oil temperature is monitored by thermocouples at three different points in the oil flow system: in the oil reservoir, and in each bearing housing. The temperature is monitored in order to satisfactorily estimate the viscosity of the lubricating oil.

The test rig is instrumented with two pairs of proximity sensors used for measuring the rotor displacement in the horizontal and vertical directions, positioned at $90^{\circ}$ in each bearing (inductive sensors "Bently Nevada"). The electric drive motor is controlled by a frequency inverter, connected to a serial gateway of the PC data acquisition, which commands the start/stop of the motor, as well as the rotation 


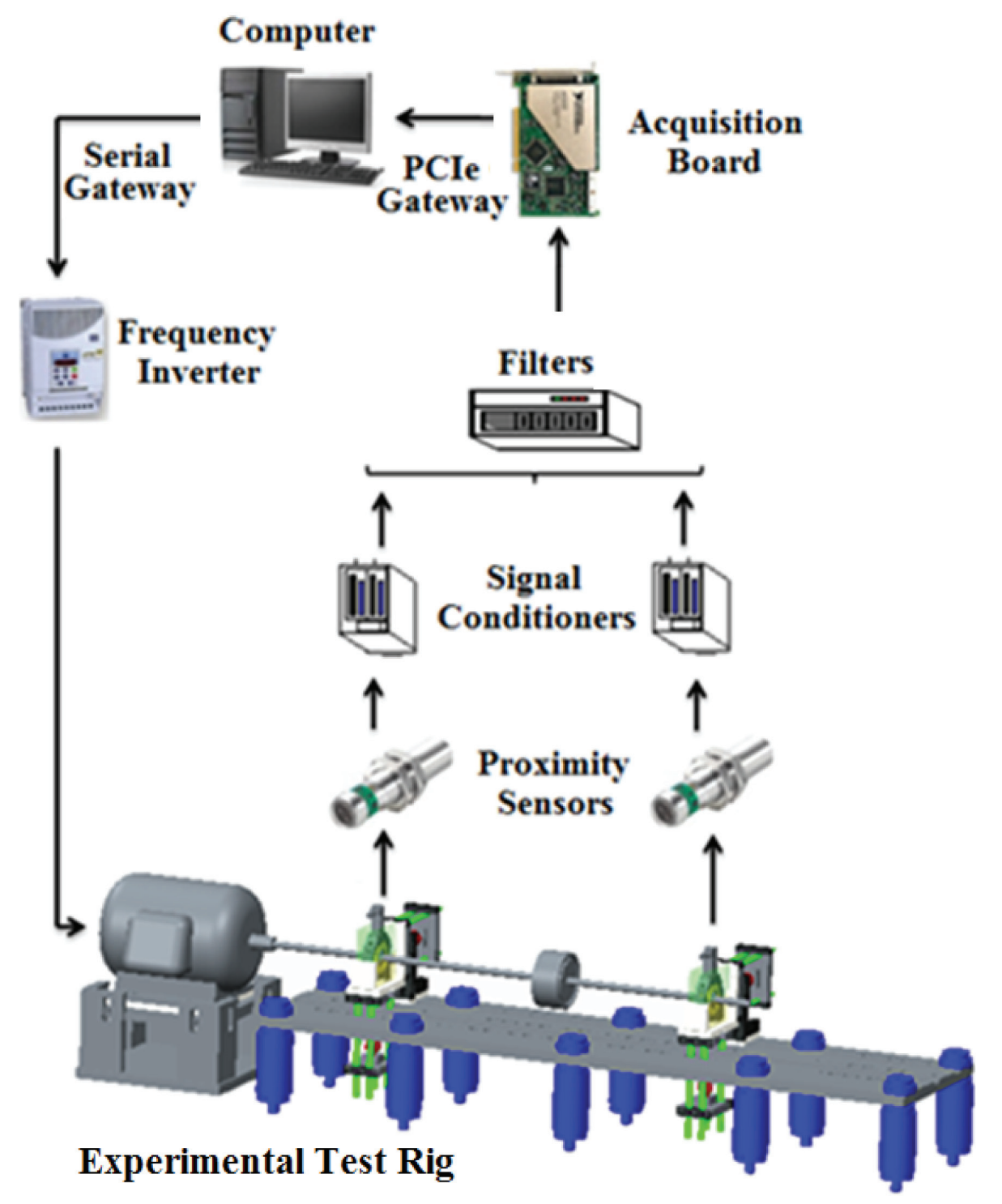

Figure 9 - Scheme of the acquisition and data processing.

frequency. The displacement signals obtained during the experiments are sent to a DC filter to remove the DC gain and to an analogic anti-aliasing filter with adjustable cut-off frequency. Finally, the data are acquired by an AT-MIO-16-E2 National Instruments ${ }^{\circledR}$ acquisition board to be processed and analyzed in the Labview ${ }^{\circledR}$ platform. Figure 9 shows an overview of the instrumentation as well as the experimental data acquisition from the test rig.

\section{EXPERIMENTAL PROCEDURE}

The experimental procedure is basically divided into two steps. The first step aims to adjust the finite element model of the rotor, as follows:

The Laval rotor is assembled on the test rig using bearings without any failure. As the tests are accomplished to obtain the unbalance frequency response, the system is balanced to estimate the residual 
unbalance moment to be considered in the numerical simulations. Then, the finite element model of the rotor is verified, that is, the number and dimensions of shaft and disc elements are adjusted.

In the second step, the bearings with the manufactured wear patterns are, then, assembled in the same rotor for an experimental evaluation of the system. In all cases, only one worn bearing is introduced in the system, in the bearing position (B2) as shown in Figure 8, while the bearing (B1) remains without wear.

\section{RESULTS AND DISCUSSION}

Initially, an adjustment of the FEM model of the rotor is made, comparing the simulated unbalance response with the experimental one obtained in the test rig. Further, the sensitivity of the bearings dynamics coefficients is analyzed. Finally, the results of the directional frequency response comparison, using different wear parameters, are presented.

\section{ADJUSTMENT OF THE FINITE ELEMENT MODEL}

In order to adjust the finite element model of the rotor, a numerical analysis of the system, based on the rotor dimensions and the bearings geometric parameters, simulates the dynamic behavior of the test rig. The adjusted model corresponds to a Laval rotor supported by two hydrodynamic bearings, as shown in Figure 10. The rotor consists of a steel shaft $\left(E=210 \mathrm{GPa}\right.$ and $\left.\rho=7800 \mathrm{~kg} / \mathrm{m}^{3}\right)$ with 13 beams elements of circular section, one disk element in the center of the rotor, two disc elements at each bearing position, representing the journal/bearings pairs, and two identical hydrodynamic bearings at nodes 3 and 11, respectively, bearing 1 (B1) and bearing 2 (B2). The excitation is given by an imposed unbalance moment of 5,5x10 $0^{-5} \mathrm{k} . \mathrm{m}$, positioned at the disk element.

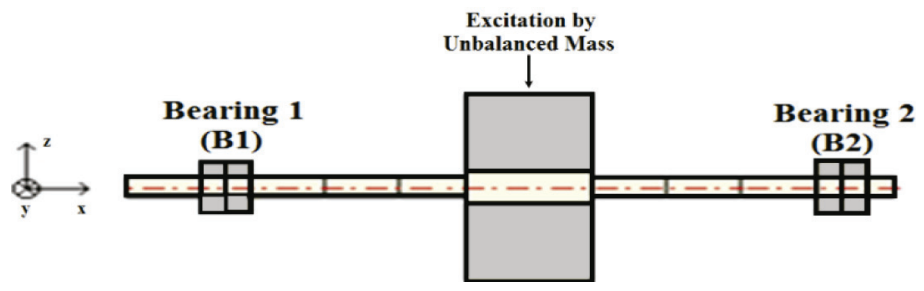

Figure 10 - Finite element model of the rotor used on the test rig.

The coefficients for the proportional structural damping are $\alpha=0$ and $\beta=1,5 \times 10^{-5}$, namely, $0.0015 \%$ of the stiffness (Liu and Novak (1995)).

Figures 11 and 12 show the frequency responses at the bearing 2 (B2), obtained with the model, overlapping with the experimental measurements, due to the known unbalance moment.

As can be seen in Figures 11 and 12, the simulated results obtained from the numerical model are very close to those obtained on the test rig, in both directions, for all the rotation frequency range. Therefore, it can be inferred that both the finite element model of the rotor, as well as the linearized model for the bearings coefficients, satisfactorily represent the experimental behavior of the test rig.

Regarding the loads on the bearings, they are evaluated considering only the gravity force; i.e., only the forces acting in the vertical direction. The loads on the bearings are measured in the test rig by load cells positioned at the bearings housing. Both static loads and locus of the shaft are obtained through a quasi-static response of the system due to the mass unbalance, i.e., avoiding the transient effects during acceleration. 


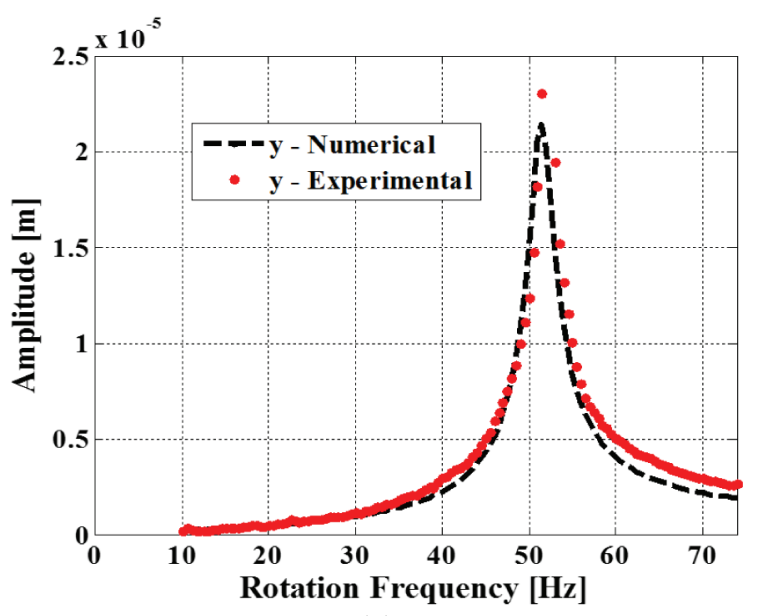

(a)

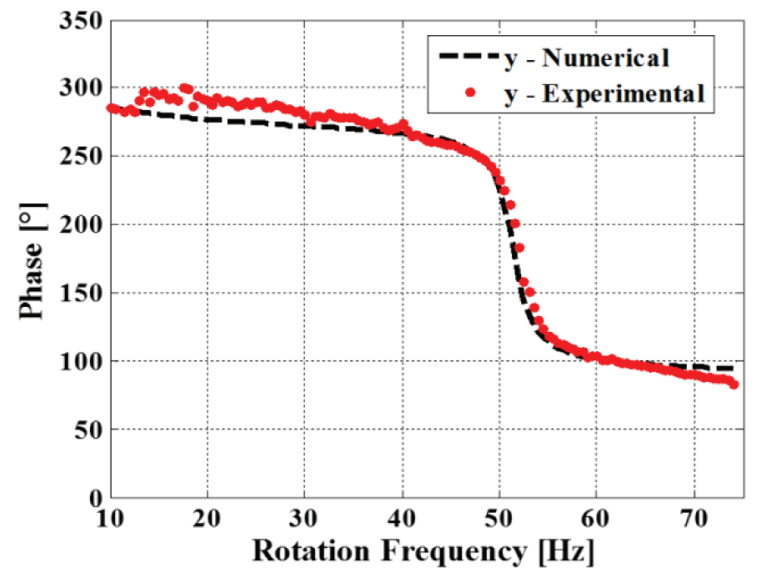

(b)

Figure 11 - Unbalanced frequency responses at Bearing 2, horizontal direction: (a) amplitude and (b) phase.

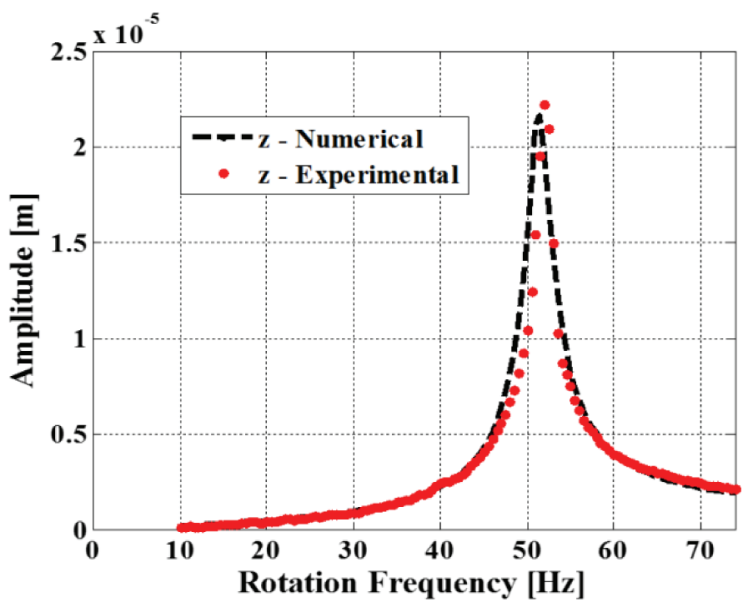

(a)

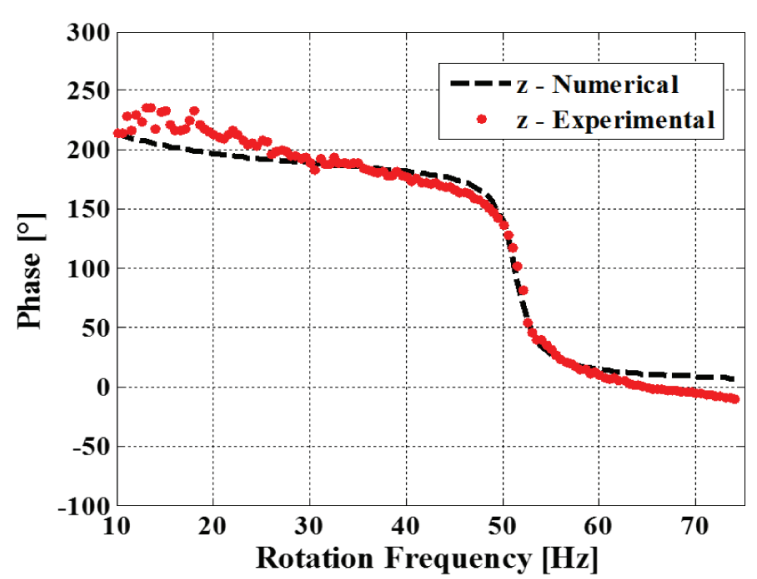

(b)

Figure 12 - Unbalanced frequency responses at Bearing 2, vertical direction: (a) amplitude and (b) phase.

From practical observation on the test rig used in the paper, the recommendation for the use of the linearization of the hydrodynamic forces is in the eccentricity ratio up to 0.6. Discrepancies are observed for larger values of eccentricities, since nonlinearities are more expressive, due to the squeeze effect in the oil film. Therefore, the tests were performed in order to maintain the eccentricity ratio in the acceptable range for the linearization of the bearings dynamic coefficients. Moreover, the authors are already working on the worn bearing model for using in the time domain, where the hydrodynamic forces will be inserted directly in the equation of motion of the rotor-bearing system, where all nonlinearities present in the model can be observed, since no linearization is required.

\section{SENSITIVITY OF THE BEARINGS DYNAMICS COEFFICIENTS}

The sensitivity of the linearized model of the bearings dynamic coefficients was evaluated before the experimental validation of the wear model through the directional response of the rotor-bearing system. Since the system of Figure 10 is practically symmetric, the main source of anisotropy is the fluid film's 
anisotropic stiffness and damping coefficients. Consequently, the diagrams with the variation of the stiffness and damping coefficients as function of the Sommerfeld number are presented for the three cases of wear configuration that are latter validated in the paper. The Sommerfeld number is presented in equation (13) and its range correspond to the operation of the experimental test-rig (from 40 to $65 \mathrm{~Hz}$ ).

$$
S=\mu \cdot\left(\frac{\pi \cdot d \cdot n}{P}\right) \cdot\left(\frac{d / 2}{C r}\right)^{2}
$$

where $\mu$ is lubricant absolute viscosity, $P$ is the bearing load, $d$ is the bearing diameter, $C r$ is the bearing radial clearance and $n$ is the rotational frequency. Figures 13 and 14 present the coefficients sensitivity to the wear depth and angular position.

From Figure 13 and 14, the changes in the direct and the cross-couple stiffness and damping coefficients can be noted in the presence of wear, through all the range of the Sommerfeld number, in both directions (Y, $Z$ ), i.e., the wear affects the anisotropic characteristic of all these coefficients. More details of the influence of the wear parameters in the pressure distribution and in the dynamic coefficients can be seen in Machado and Cavalca (2015).

\section{DIRECTIONAL RESPONSE OF THE ROTOR-BEARING SYSTEM}

This section deals with the comparison of the directional frequency response (DFR) of the rotating system for three different wear configurations, varying its depth and angular position. The worn bearings were

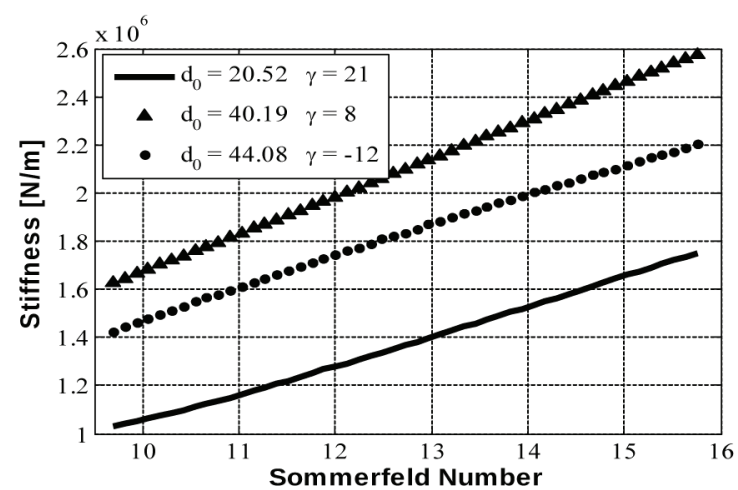

(a)

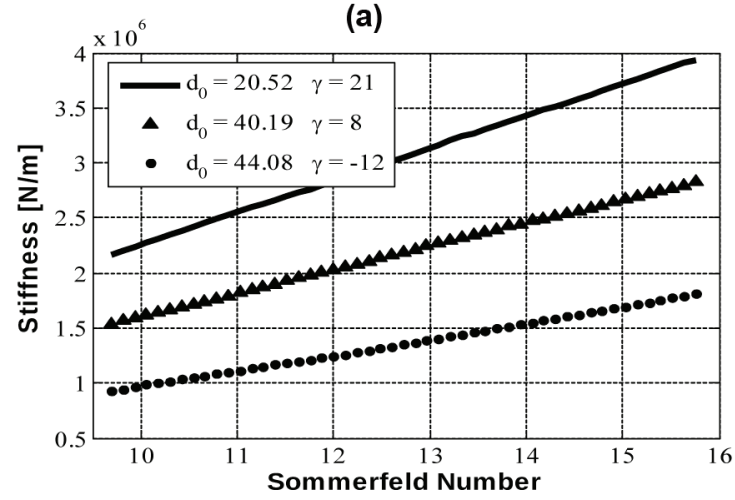

(c)

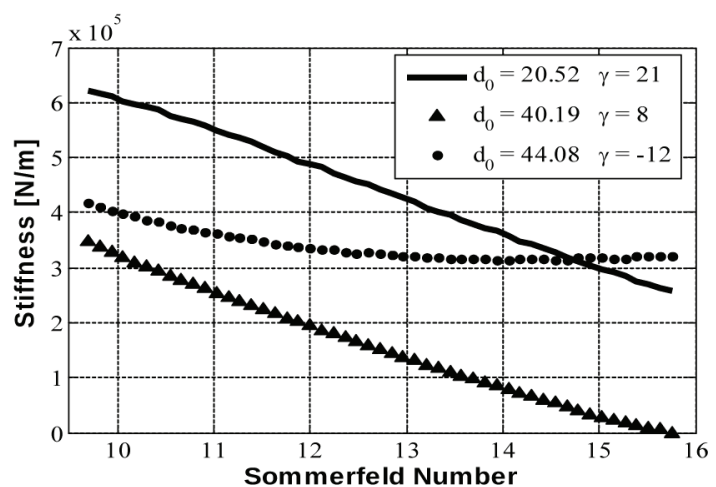

(b)

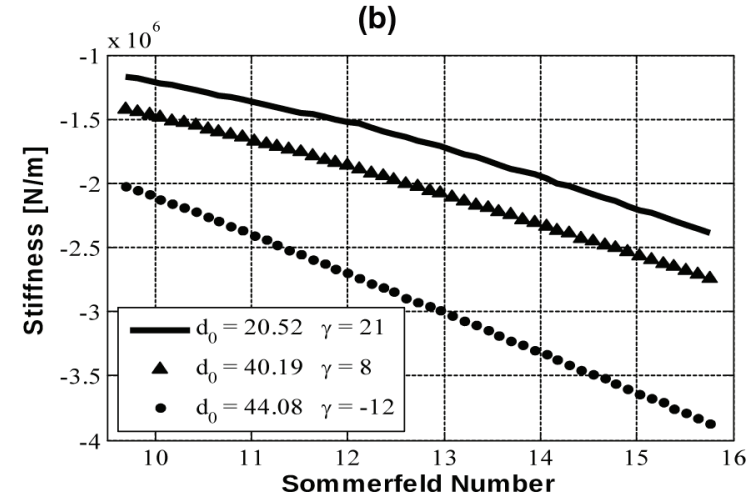

(d)

Figure 13 - Stiffness coefficients - (a) $K_{y y}$, (b) $K_{z z}$, (c) $K_{y z}$ and (d) $K_{z y}$. 


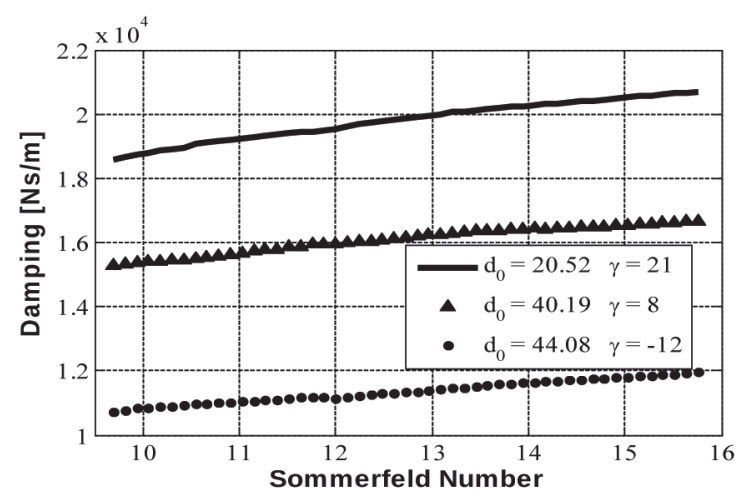

(a)

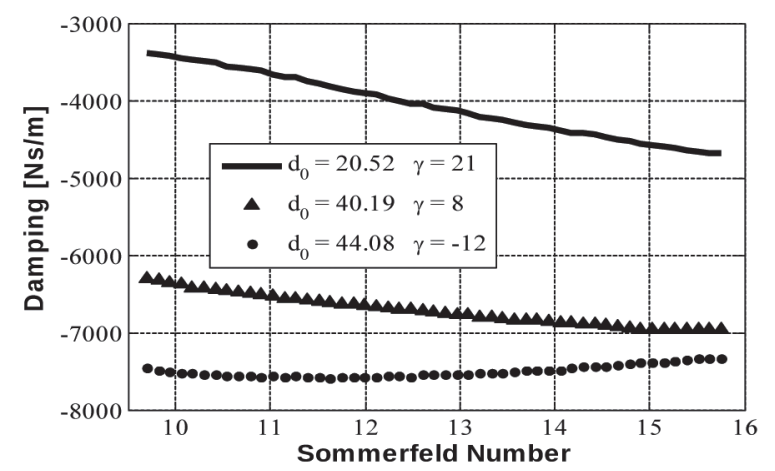

(c)

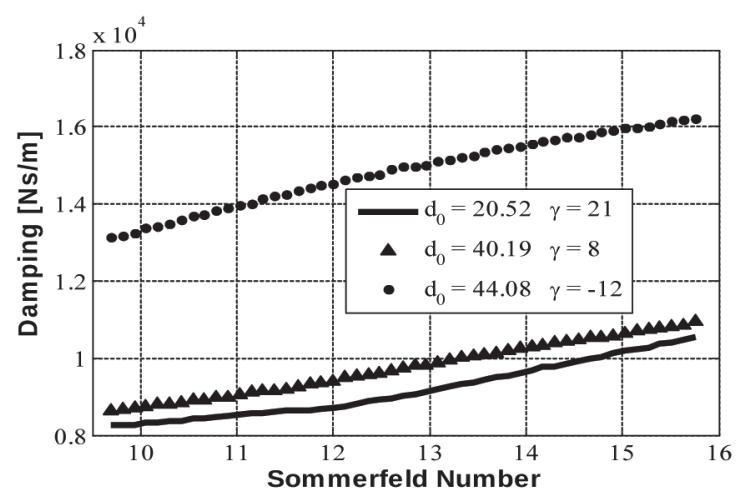

(b)

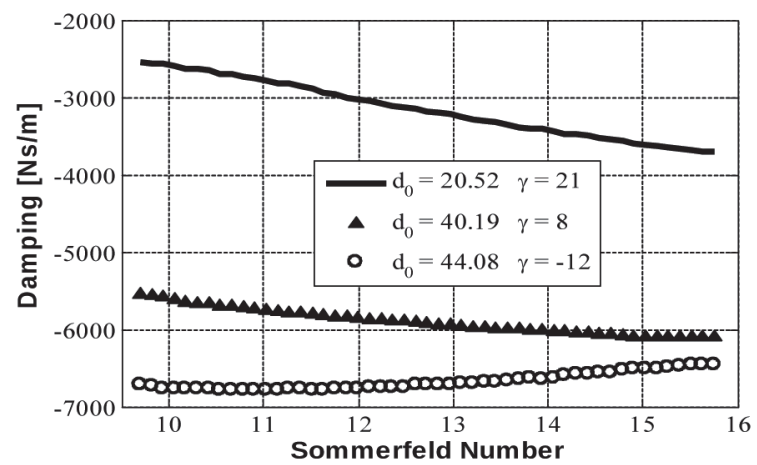

(d)

Figure 14 - Damping coefficients - (a) $R_{y y}$, (b) $R_{z z}$, (c) $R_{y z}$ and (d) $R_{z y}$.

manufactured according to the limitations of the workshop tools. Table I shows the wear parameters manufactured on the bearings.

In all cases, the system had only one worn bearing, bearing (B2), farther of the drive motor. Both simulated and experimental responses were evaluated for the worn bearing node at a frequency range from 40 to $65 \mathrm{~Hz}$, with steps of $0.5 \mathrm{~Hz}$ (around the first natural frequency of the system), and the amplitude is shown in the form ' 0 to peak'.

TABLE I

Manufactured bearings wear characteristics.

\begin{tabular}{ccc}
\hline Cases & Maximum depth $[\boldsymbol{\mu m}]$ & Angular position $\left[{ }^{\circ}\right]$ \\
\hline 1 & 20.52 & 21 \\
\hline 2 & 44.08 & -12 \\
\hline 3 & 40.19 & 8 \\
\hline
\end{tabular}

Figure 15 presents both experimental and simulated DFRs for the system with bearings with no wear and Figures 16 to 18 the same results for the three cases of Table I for the worn bearing position (bearing (B2)).

It can be noted that the simulated directional frequency responses show a good agreement with the experimental responses, demonstrating the potential of the modified wear model in represent satisfactorily the experimental DFRs curves in the frequency range where the numerical model was validated. In Figure 


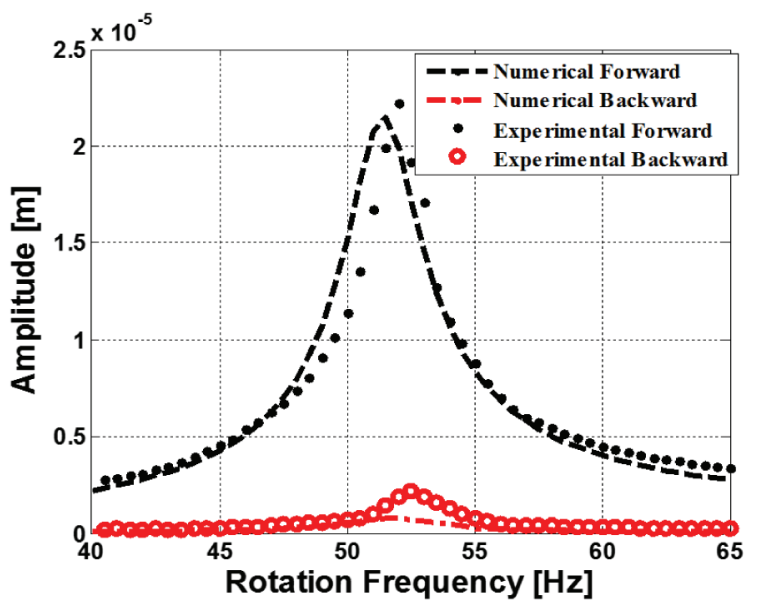

(a)

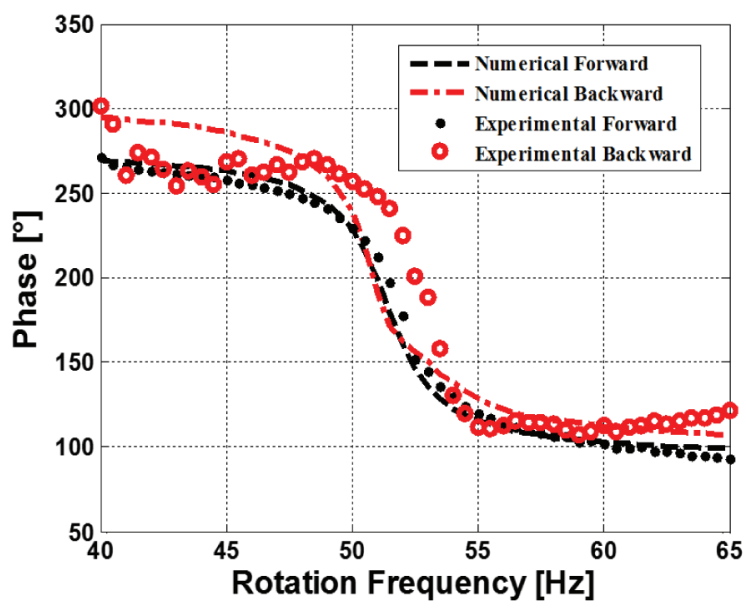

(b)

Figure 15 - Directional response of the system (bearing B2 position) for bearings with no wear - a) Amplitude and b) Phase.

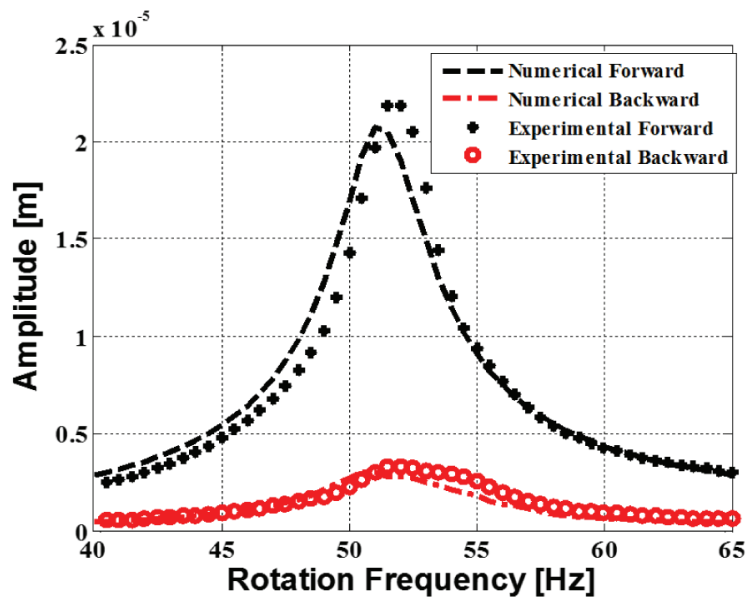

(a)

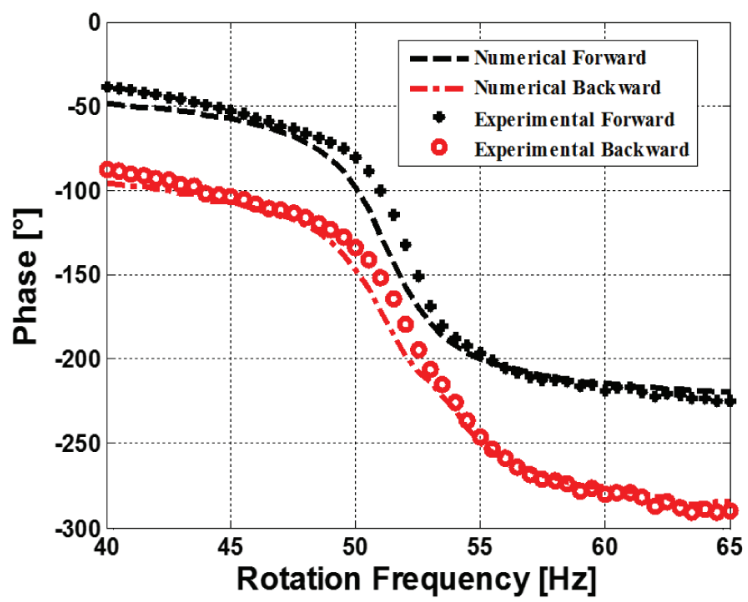

(b)

Figure 16 - Directional response of the system (bearing B2 position) for Case 1 - a) Amplitude and b) Phase.

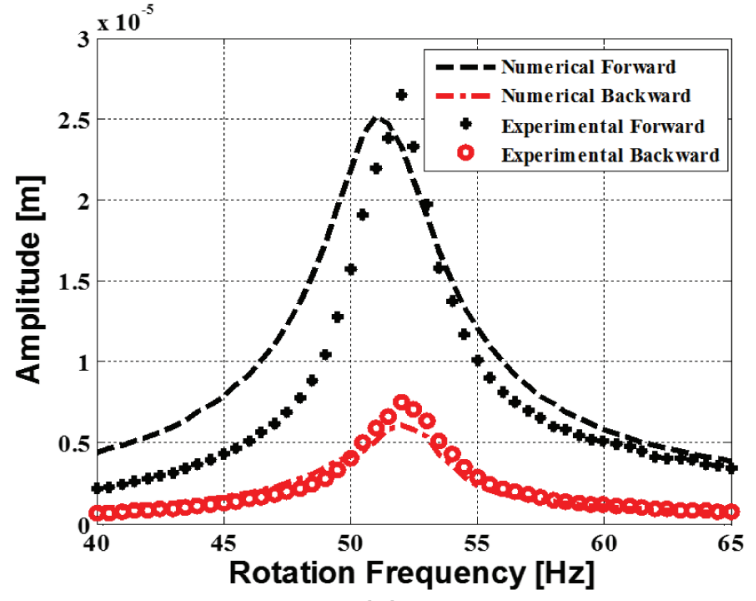

(a)

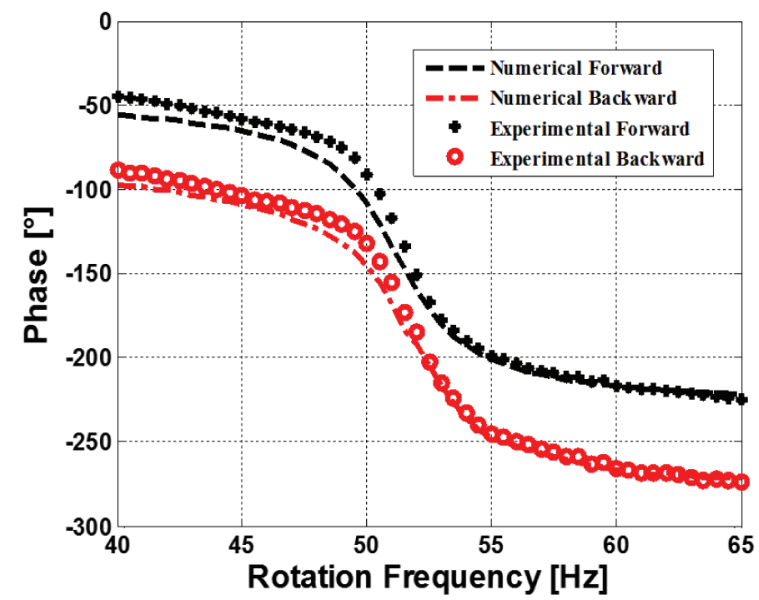

(b)

Figure 17 - Directional response of the system (bearing B2 position) for Case 2 - a) Amplitude and b) Phase. 


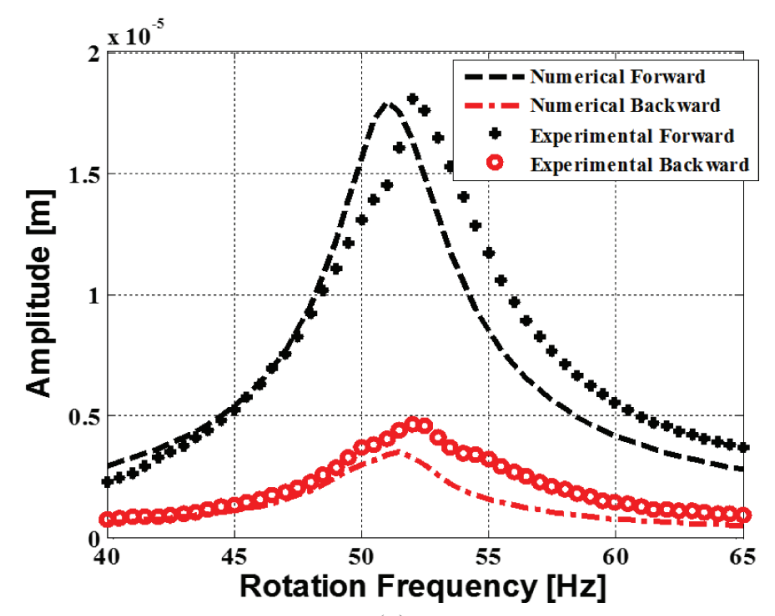

(a)

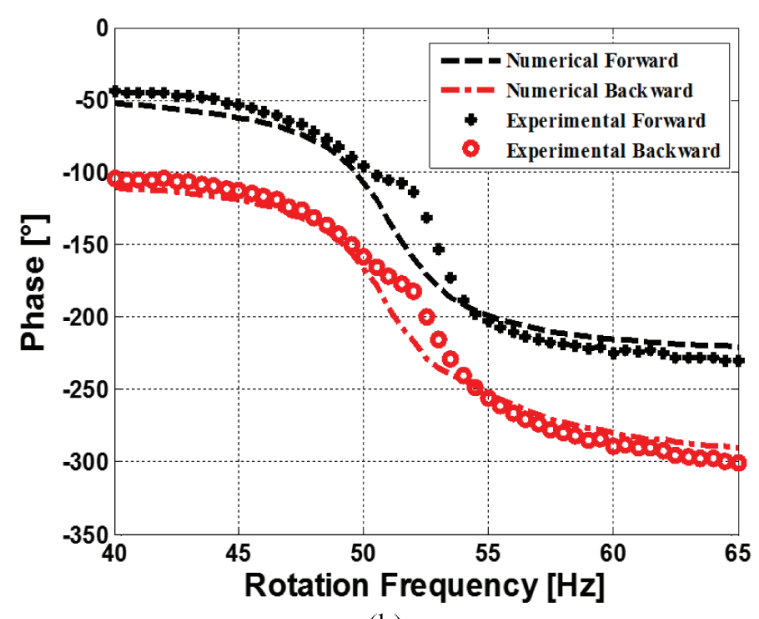

(b)

Figure 18 - Directional response of the system (bearing B2 position) for Case 3 - a) Amplitude and b) Phase.

15 the presence of the backward mode can be observed for the bearing with no wear. This is due to the fact that even a smooth cylindrical bearing has some degree of anisotropy in the equivalent dynamic coefficients. Moreover, in the simulated results and the experimental tests, there is a significant growth on the backward precession component as the wear depth is increased, showing that the DRFs are really sensitive to the wear model.

\section{CONCLUSIONS}

In this paper, a numerical model (previously proposed by the authors) to represent the main parameters of a worn bearing was discussed and an experimental apparatus was assembled to measure the dynamic response of the system, in order to validate the model.

First, the algorithm for calculating the directional frequency response of the entire rotating system was tested. It was found that the model satisfactorily represent the experimental system behavior of the test rig. After that, three different wear configurations, varying the depth and the angular position, were tested in the test rig and also in the numerical model. Then a comparison of the directional frequency response (DFR) of the rotating system was made in all cases.

The simulated directional frequency responses showed a good agreement with the experimental responses, demonstrating the potential of the proposed wear model in satisfactorily represent the experimental DFRs curves in the frequency range where the numerical model was validated. Moreover, in the simulated results and experimental tests, there is a significant growth of the backward precession component as the wear depth is increased, showing that the DRFs are really sensitive to the wear model proposed in this work.

\section{ACKNOWLEDGMENTS}

The authors thank Fundação de Amparo à Pesquisa do Estado de São Paulo (FAPESP), Coordenação de Aperfeiçoamento de Pessoal de Nível Superior (CAPES) and Conselho Nacional de Desenvolvimento Científico e Tecnológico (CNPq) for the financial support of this research. 


\section{REFERENCES}

ARGHIR M, ALSAYED A AND NICOLAS D. 2002. The finite volume solution of the Reynolds equation of lubrication with film discontinuities. Int J Mech Sci 44: 2119-2132.

CHASALEVRIS A, NIKOLAKOPOULOS P AND PAPADOPOULOS C. 2013. Dynamic effect of bearing wear on rotor-bearing system response. ASME J Vib Ac 135(1): 11008-11020.

DUFRANE KF, KANNEL JW AND MCCLOSKEY TH. 1983. Wear of steam turbine journal bearings at low operating speeds. J Lub Tech 105: 313-317.

FILLON M AND BOUYER J. 2004. Thermohydrodynamic analysis of a worn plain journal bearing. Trib Int 37(2): 129-136.

GERTZOS KP, NIKOLAKOPOULOS PG, CHASALEVRIS AC AND PAPADOPOULOS CA. 2011. Wear identification in rotorbearing systems by measurements of dynamic bearing characteristics. Comp Struc 89: 55-66.

HASHIMOTO H, WADA S AND NOJIMA K.1986. Performance characteristics of worn journal bearings in both laminar and turbulent regimes. Part I: Steady-state characteristics. ASLE Trans 29: 565-571.

KATZENMEIER G. 1972. The influence of materials and surface quality on wear behaviour and load capacity of journal bearings. In: Proc Inst Mech Eng Trib Conv: 80-84.

KRAMER E. 1993. Dynamics of rotors and foundations. Springer-Verlag, New York, 381 p.

KUMAR A AND MISHRA S. 1996a. Stability of a rigid rotor in turbulent hydrodynamic worn journal bearings. Wear 193: 25-30.

KUMAR A AND MISHRA S. 1996b. Steady-state analysis of non-circular worn journal bearings in non-laminar lubrication regimes. Trib Int 29: 493-8.

LALANNE M AND FERRARIS G. 1998. Rotordynamics Prediction in Engineering. J Wiley \& Sons, England, 266 p.

LEE CW. 1991. A Complex Modal Testing Theory for Rotating Machinery. Mech Sys Sig Proc 5: 119-137.

LIU W AND NOVAK M. 1995. Dynamic behaviour of turbine-generator-foundation. Earth Eng Struc Dyn 24: 339-360.

MACHADO TH AND CAVALCA KL. 2011. Dynamic analysis of cylindrical hydrodynamic bearings with geometric discontinuities. In: Int Conf Vib Prob ICoVP-2011, Prague, Czech Republic: 1-10.

MACHADO TH AND CAVALCA KL. 2015. Modelling of hydrodynamic bearing wear in rotor-bearing systems. Mech Res Com 69: 15-23.

MOKHTAR M, HOWARTH R AND DAVIES P. 1977. Wear characteristics of plain hydrodynamic journal bearings during repeated starting and stopping. ASLE Trans 20: 191-194.

NELSON HD AND MCVAUGH JM.1976. The dynamics of rotor-bearing systems using finite elements. ASME J Eng Ind 98(2): 593-600.

PAPADOPOULOS CA, NIKOLAKOPOULOS PG AND GOUNARIS GD. 2008. Identification of clearances and stability analysis for a rotor-journal bearing system. Mech Mach Theo 43: 411-426.

SCHARRER JK, HECHT RF AND HIBBS RI. 1991. The effects of wear on the rotordynamic coefficients of a hydrostatic journal bearing. ASME J Trib 113: 210-213.

SUZUKI K AND TANAKA M. 1995. Stability characteristics of worn journal bearing. In: Proc Asia-Pacific Vib Conf, Kuala Lumpur: 296-301.

VAIDYANATHAN K AND KEITH TG. 1991. Performance characteristics of cavitated noncircular journal bearings in the turbulent flow regime. Trib Trans 34: 35-44.

WU T, MAO J, DONG G, XU H AND XIE Y. 2008. Journal bearing wear monitoring Via on-line Visual Ferrograph. Adv Mater Res 44: 189-194. 